Case Report

\title{
Orientia tsutsugamushi and Epstein-Barr virus in a young adult with acalculous cholecystitis: a case report and epidemiology of scrub typhus in the Maldives
}

\author{
Hisham Ahmed Imad ${ }^{1,2 *}$, Aishath Azna Ali ${ }^{3}$, Mariyam Nahuza ${ }^{3}$, Rajan Gurung ${ }^{3}$, Abdulla Ubaid ${ }^{3}$, Aishath Maee- \\ sha $^{4}$, Sariu Ali Didi ${ }^{4}$, Rajib Kumar Dey ${ }^{4}$ Abdullah Isneen Hilmy ${ }^{4,5}$, Aishath Hareera ${ }^{6}$, Ibrahim Afzal ${ }^{6}$, Wasin \\ Matsee $^{7}$, Wang Nguitragool ${ }^{1,8}$, Emi. E. Nakayama ${ }^{2}$ and Tatsuo Shioda ${ }^{2}$
}

1 Mahidol Vivax Research Unit, Faculty of Tropical Medicine, Mahidol University, Bangkok 10400, Thailand. (W.N.) wang.ngu@mahidol.edu

2 Department of Viral Infections, Research Institute for Microbial Diseases, Osaka University, Osaka, Japan. (E.E.N.); emien@biken.osaka-u.ac.jp, (T.S.); shioda@biken.osaka-u.ac.jp

3 Department of Surgery, Indira Gandhi Memorial Hospital, Malé 20002, Maldives. (A.A.A); dr.azna@igmh.gov.mv, (M.N.); dr.mariyamnahuza@igmh.gov.mv, (R.G.); dr.rajangurung@igmh.gov.mv, (A.U.); dr.ubaid@igmh.gov.mv,

4 Department of Medicine, Indira Gandhi Memorial Hospital, Malé 20002, Maldives. (A.M.); dr.aishathmaeesha@igmh.gov.mv, (S.A.D.); sariualididi@igmh.gov.mv, (R.K.D.); dey@igmh.gov.mv, (A.I.H.) hlmabd001@myuct.ac.za

5 Gastrointestinal Unit, Department of Medicine, Groote Schuur Hospital, University of Cape Town 7935, South Africa.

6 Health Protection Agency, Ministry of Public Health, Malé 20002, Maldives. (A.H.); hareera@health.gov.mv, (I.A.); afzal@health.gov.mv

7 Department of Clinical Tropical Medicine, Faculty of Tropical Medicine, Mahidol University, Bangkok 10400, Thailand. (W.M.); wasin.mat@mahidol.edu

8 Department of Molecular Tropical Medicine and Genetics, Faculty of Tropical Medicine, Mahidol University, Bangkok 10400, Thailand

* Correspondence: hishamahmed.ima@mahidol.ac.th, or imad@biken.osaka-u.ac.jp, Tel.: (+66631501402)

\begin{abstract}
Scrub typhus is a neglected tropical disease predominantly occurring in Asia. The causative agent is a bacterium transmitted by the larval stage of mites found in rural vegetation in endemic regions. Cases of scrub typhus frequently present as acute undifferentiated febrile illness, and without early diagnosis and treatment, the disease can develop fatal complications. We retrospectively reviewed de-identified data from a 23-year-old woman who presented to an emergency department with complaints of worsening abdominal pain. On presentation, she appeared jaundiced and toxic-looking. Other positive findings on abdominal examination were a positive Murphey's sign, abdominal guarding and hepatosplenomegaly. Magnetic resonance cholangiopancreatography demonstrated acalculous cholecystitis. Additional findings included eschar on the medial aspect of the left thigh with inguinal regional lymphadenopathy. Further, positive results were obtained for immunoglobulins $\mathrm{M}$ and $\mathrm{G}$, confirming scrub typhus. Workups for other infectious causes of acute acalculous cholecystitis detected human herpesvirus 4 (Epstein-Barr virus). Whether that represented acute infection or re-activation of the Epstein-Barr virus could not be determined. As other reports have described acute acalculous cholecystitis in adult scrub typhus patients, we recommend doxycycline to treat acute acalculous cholecystitis in endemic regions while awaiting serological confirmation.
\end{abstract}

Keywords: acute acalculous cholecystitis; Orientia tsutsgugamushi; scrub typhus; eschar; Epstein-Barr virus; re-activation; clinical manifestation; Maldives 


\section{Introduction}

Infectious diseases are well-known as a scourge of wars. Some infamous accounts include endemic typhus during the Franko-Russo war, trench fever in World War I, and scrub typhus during World War II [1]. The latter of these, scrub typhus, is caused by the rickettsial species Orientia tsutsugamushi, an intra-cytosolic coccobacillus with three main prototype strains (Karp, Gilliam and Kato) that can inflict disease of varying severities in humans [2].

The earliest description of scrub typhus appears to be an account of "shashitsu" in Chinese literature from 313 AD [3]. Centuries later, the word "tsutsugamushi" was used in Japan to describe a febrile illness following insect exposures. Tsutsugamushi was a feverish delirium associated with regional lymphadenopathy, rash and a pathognomonic cutaneous finding of tache noire or black spot, commonly referred to now as eschar [4].

The skin lesion of eschar is characterized by central necrosis and surrounding erythema with a halo sign [5]. The necrosis results from acidic enzymes channeled through a stylosome, a funnel created by the vector, gaining access via a skin pore or hair follicle [6]. This triggers a host response comprising infiltration of white blood cells into the dermis, causing the peripheral crusting of the scab.

In the Asia-Pacific theater of World War II, stringent vector control measures were employed to protect deployed troops from the parasitic larval stage of a chigger-mite found amongst the vegetation in endemic regions $[7,8]$. Nevertheless, this task proved challenging during the pre-antibiotic era and case fatality reached up to $40 \%$. In 1947 , the first cases of successful treatment with chloromycetin were reported from the Malay Peninsula $[9,10]$.

However, despite modern availability of surefire antibiotics with bacteriostatic properties that stalled the bacterium O. tsutsugamushi and allowed phagocytes to clear up the infection, the disease still occurred after exposure to the vector. Escalating this problem is the geographic expansion of previously restricted endemic regions, and cases clinically compatible with scrub typhus or showing serological evidence have been reported from Chile, the United Arab Emirates and parts of Africa [11-18].

The Maldives is one endemic region for scrub typhus in Asia [5]. The archipelago has a peculiar historical account comprising a six-decade period with absence of the disease ended by re-emergence with 168 cases, including 10 fatalities [19]. Since that re-emergence in the Maldives, sentinel reporting of scrub typhus has shown occurrences throughout the year, with two periodic peaks observed during holiday seasons [20].

In tropical regions, coinfections with other tropical diseases are often observed, obfuscating the clinical characteristics of diseases[21]. This provides a major impediment to correct clinical judgment and often results in a poor clinical trajectory with missed diagnoses[22]. It has been previously reported that $15 \%$ of scrub typhus cases had coinfection with other diseases [23]. Complications often involve multiple systems and fatalities are often due to multi-organ dysfunction syndrome [24]. Commonly involved systems include the respiratory, central nervous, cardiovascular, renal, and gastrointestinal systems. However, reports of complications such as pancreatitis, acute cholecystitis, or acalculous cholecystitis in scrub typhus are rare.

First described in 1844 by a surgeon, acute acalculous cholecystitis (AAC) occurs in mainly critically ill geriatric patients. AAC also occurs postoperatively, and in patients requiring medical interventions such as mechanical ventilation or prolonged parenteral nutrition and fasting $[25,26]$. This pathology accounts for $10 \%$ of all cases of acute cholecystitis and is associated with high morbidity and mortality rates [27, 28]. The pathophysiology involves inflammation of the gallbladder causing bile stasis and disruption of circulation causing ischemia and necrosis or rupture of the gallbladder, which then involves the peritoneum. In such cases, AAC is associated with infectious etiologies, mainly involving Gram-negative Enterobacteriaceae or Gram-positive spore-forming bacilli [29]. Interestingly, some other pathogens like Epstein-Barr virus and O. tsutsugamushi have been described to be associated with AAC in otherwise healthy 
adults [30-41]. Here we report a case of acute acalculous cholecystitis in a young healthy adult from the Maldives.

\section{Materials and Methods}

The case we described here presented to Indira Gandhi Memorial Hospital (IGMH) in Malé, Republic of Maldives, in July 2021. De-identified clinical and laboratory data during the hospitalization were reviewed using in-patient medical charts. Diagnostics for O. tsutsugamushi included the "Scrub Typhus Detect ${ }^{\mathrm{TM}}$ " immunoglobulin (Ig)M/IgG enzyme-linked immunosorbent assay (InBios International, Seattle, WA, USA). Diagnostics for Epstein-Barr virus included VIDAS®EBV which detected Epstein-Barr viral capsid antigen (EBV-VCA) IgM/IgG, early antigen (EBV-EA) IgG, and nuclear antigen (EBV-NA) IgG by chemiluminescence assays (bioMérieux Italia, Florence, Italy). Hemoculture was performed using an automated culture system (bioMérieux, Durham, NC, USA). We also obtained epidemiological surveillance data for the preceding nine years (2012-2021) on scrub typhus in the Maldives from the Health Protection Agency at the Ministry of Health.

\section{Case report}

We present the case of a previously healthy 23-year-old woman who presented to the emergency department of IGMH with worsening abdominal pain and fatigue (Figure S1). The presenting complaint was associated with a 9-day history of high-grade fever accompanied by chills, and displaying a diurnal pattern. Four days before presentation, the patient had consulted a primary-care physician and had been prescribed amoxicillin and clavulanic acid for tonsillopharyngitis. Additional history included several episodes of vomiting with the onset of colicky, non-radiating abdominal pain of severe intensity, without any apparent aggravating or relieving factors. The patient had traveled from her home island of Gadhdhoo, in a southern atoll in the Maldives, to the capital city of Malé to visit friends and relatives a couple of days before falling ill, as illustrated in Figure S2. The annual descriptive epidemiology of scrub typhus in the Maldives is presented in Figure S3.

On examination, the patient was conscious and coherent, but appeared dehydrated, jaundiced, and toxic-looking. Oral temperature was $39^{\circ} \mathrm{C}$, and heart rate was 110 beats/min with a mean arterial blood pressure of $73 \mathrm{mmHg}$. Both tonsils were enlarged with evidence of pharyngitis and palpable cervical lymphadenopathy. Evaluations of the cardiovascular, respiratory, and central nervous systems found no abnormalities. Other abdominal examinations revealed a positive Murphy's sign and guarding on palpation of the abdomen. The results of laboratory investigations on presentation are shown in Table 1. 
Table 1. Laboratory results at presentation and before discharge.

\begin{tabular}{|c|c|c|}
\hline Day of illness & 9 & 14 \\
\hline Leukocyte/ $\mu \mathrm{L}$ & 6,650 & 8,030 \\
\hline Neutrophils/ $\mu \mathrm{L}$ & 4,788 & 4,496 \\
\hline Lymphocytes & 14,616 & 16,240 \\
\hline Monocytes/ $\mu \mathrm{L}$ & 1,340 & 3,265 \\
\hline Eosinophils/ $\mu \mathrm{L}$ & 0 & 37.5 \\
\hline Basophils/ $/ \mu \mathrm{L}$ & 0 & 0 \\
\hline Platelets/ $\mu \mathrm{L}$ & 269,000 & 545,000 \\
\hline Hemoglobin $(\mathrm{g} / \mathrm{dL})$ & 10.6 & 7.8 \\
\hline Hematocrit (\%) & 32.9 & 24.6 \\
\hline Total Bilirubin (mg/dL) & 4.7 & 3.5 \\
\hline Direct Bilirubin (mg/dL) & 3.5 & 2.6 \\
\hline Total protein $(\mathrm{g} / \mathrm{dL})$ & 6.9 & 6.8 \\
\hline Albumin (g/dL) & 3.4 & 2.8 \\
\hline Alkaline phosphatase (IU/L) & 288 & 250 \\
\hline Aspartate aminotransferase (IU/L) & 140 & 76 \\
\hline Alanine aminotransferase (IU/L) & 157 & 93 \\
\hline Creatinine $(\mathrm{mg} / \mathrm{dL})$ & 0.8 & 0.6 \\
\hline Urea (mg/dL) & 10 & 6.4 \\
\hline CRP (mg/dL) & 7.7 & 2.8 \\
\hline Sodium (mmol/L) & 131 & 134 \\
\hline Potassium (mmol/L) & 4.5 & 4.3 \\
\hline Ferritin $(\mathrm{ng} / \mathrm{mL})$ & 1,299.3 & \\
\hline LDH (IU/L) & 726 & \\
\hline EBV-VCA IgM (IU/mL) & 63.6 & \\
\hline EBV-VCA IgG (IU/mL) & 89.9 & \\
\hline EBV-EA IgG (IU/mL) & 16.1 & \\
\hline EBV-NA IgG (IU/mL) & 574.0 & \\
\hline Hemoculture & & no growth \\
\hline Orientia tsutsugamushi IgM & positive & \\
\hline Orientia tsutsugamushi IgG & positive & \\
\hline
\end{tabular}

CRP: C-reactive protein, LDH: lactate dehydrogenase, EBV-VCA: Epstein-Barr virus viral capsid antigen, EBV-EA; Epstein-Barr virus early antigen, EBV-NA: Epstein-Barr virus nuclear antigen, IgM: immunoglobulin M, IgG: immunoglobulin G.

The initial hematological profile on presentation showed lymphocytosis and increased monocytes, although leukocytes, neutrophils, and platelets remained within normal ranges. Hyperbilirubinemia and elevated transaminases were identified from liver function tests. Further, hyperferritinemia and elevated levels of C-reactive protein were identified. Ultrasonography confirmed Murphey's sign from the probe test and demonstrated edema of the gallbladder wall. Computed tomography (CT) of the abdomen revealed hepatosplenomegaly. Magnetic resonance cholangiopancreatography subsequently confirmed acalculous cholecystitis, as shown in Figure 1. 

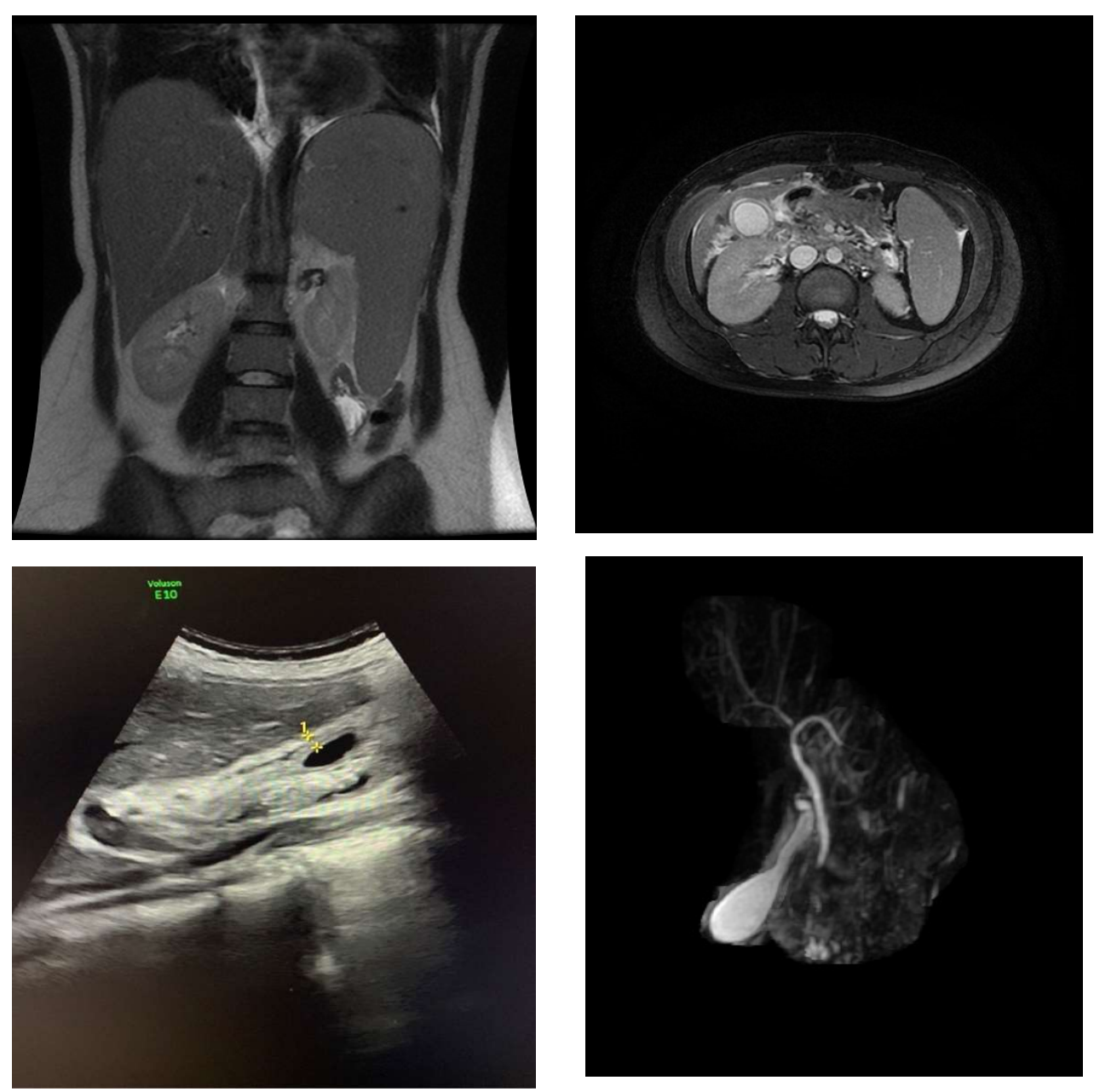

(c)

(d)

Figure 1. Imaging of the abdomen during hospitalization. a) Coronal-view CT of the abdomen shows hepatosplenomegaly. b) Axial-view CT of the abdomen demonstrates cholecystitis with enhancement of the gallbladder wall. c) Longitudinal ultrasonography depicts an edematous gallbladder wall (wall thickness $>4 \mathrm{~mm}$ ) without lithiasis. d) Magnetic resonance cholangiopancreatography reveals a ballooned gallbladder without any ductal obstruction, but with signs of inflammation consistent with acalculous cholecystitis.

The patient was admitted with parenteral fluids to correct dehydration, broad-spectrum antibiotics (cefotaxime at $2 \mathrm{~g} /$ day, metronidazole at $1500 \mathrm{mg} /$ day), and parenteral analgesics. During nursing care, eschar was noticed on the medial aspect of the left thigh (Figure S5). Left regional inguinal lymphadenopathy was also detected. Doxycycline was started at a loading stat dose of $200 \mathrm{mg}$ followed by $200 \mathrm{mg} / \mathrm{day}$ for seven days. Fever cleared within $52 \mathrm{~h}$ of beginning doxycycline (Figure S6). Symptoms of abdominal pain gradually improved, and at the time of discharge from hospital, she had residual signs of fatigue.

\section{Discussion}

The first description of scrub typhus from the Maldives was reported during World War II [8]. By mid-1944, when the momentum in the war had shifted away from the battlefront following the historic invasion at Normandy, several hundred deployed British troops in the southern atolls of the Maldives fell victim to scrub typhus [42]. This prompted several entomological expeditions to the hotspot atolls identified in the Mal- 
dives, such as Gaaf atoll, and the vector and reservoir local to the islands were identified $[43,44]$. Reservoir eradication proved effective, with no subsequent cases reported. Scrub typhus was then simply forgotten until its sudden re-emergence in $2002[5,19,45]$. Since decades of sentinel data reflect the existence of O. tsutsugamushi throughout the archipelago, reports of scrub typhus from Faafu, Vaavu, and Meemu atolls are recent. We loosely associate this peculiar finding with urbanization, which could have exposed individuals to areas of vegetation where the vector is found [5].

The incubation of scrub typhus is 6-21 days [24]. Consequently, when obtaining a travel history, a minimum of three weeks is necessary to consider scrub typhus among the differential diagnoses. The patient in the present case was from an island within a hotspot for scrub typhus in the Maldives. However, she did not recall any particular activities that might have exposed her to the vector, such as lying on grassy vegetation or exploring tropical forest without proper preventive measures (such as use of insecticides, or covering exposed skin with long-sleeved clothes to avoid contact with insects). Nevertheless, the patient did confirm walking down a trail in a forest to get to the beach. The particular trails are narrow, with fringes of vegetation along both sides to the sandy beach. Interestingly, the patient also acknowledged knowing about the disease on the island through folklore of individuals developing fever after returning from the woods, and the eschar was locally referred to as "Gadhdhoo bihi", translating as "skin lesion from the island of Gadhdhoo". This reflects historical accounts of the endemicity of scrub typhus in the region.

Clinical manifestations of scrub typhus commonly appear first as undifferentiated febrile illness. During the early phase of disease, systemic symptoms are predominantly present without localizing foci of infection. Nevertheless, our patient presented with intermittent fever and she had complaints of sore throat and documented findings of tonsillopharyngitis from her initial visit to the primary-care physician. Similar manifestations had previously been described in scrub typhus patients, where misdiagnosis unfortunately led to complications from scrub typhus [46]. The exact cause of the intermittent fever observed in scrub typhus is unknown, but is likely to involve a pyrogen and countereffective cryogen production. Here, we assumed the exogenous pyrogen to be the peptidoglycan-like structure of O. tsutsugamushi and the cryogen to be interleukin 10 (IL-10) expressed by host cells [47-50]. Expression of IL-10 is one of the mechanisms utilized in immune evasion by the bacterium [51, 52].

Gastrointestinal (GI) symptoms occur in scrub typhus, but less frequently compared to systemic symptoms [53]. GI symptoms were reported in 20-76\% of scrub typhus cases, with varying severity $[54,55]$. As described in one cohort, the most common GI symptom was nausea, followed by abdominal pain, vomiting, and diarrhea [53]. The patient in the present case developed nausea during the third day after symptoms onset and experienced several episodes of vomiting with bile contents, up until the abdominal pain started. A correlation between endoscopic findings suggestive of gastrointestinal vasculitis and disease severity has previously been demonstrated [55]. O. tsutsugamushi shows tropism to several host cells, including endothelial cells with the pathogenicity of endotheliitis [56]. Dissemination occurs within macrophages, and the bacterium has previously been detected in several organs, including in the human gallbladder [57].

Other studies have previously described hepatosplenomegaly, gallbladder or periportal edema and abdominal lymphadenopathy as GI findings visualized in patients with scrub typhus [58]. In the same cohort, splenomegaly was detected in 15 of 19 cases and $16 \%$ developed splenic infarcts due to acute enlargement of the spleen. The patient in our case presented also exhibited massive splenomegaly, but fortunately no splenic infarcts developed. In addition, the liver was enlarged. Animal studies have demonstrated hepatomegaly resulting from periportal inflammation [59]. Similarly, thickening of the gallbladder wall can result from perivasculitis or vasculitis [35]. This thickening of the gallbladder wall was detected in 9 of 19 cases previously [58]. Presentation with acute abdomen in cases of scrub typhus is rare, but does occur as in this case, and has on occasion resulted in explorative surgeries [60]. 
The patient in this case showed the triad of fever, right upper quadrant pain, and clinical jaundice, suggestive of acute cholecystitis. Nevertheless, some hematological features like leukocytosis were absent from the hematological profile. In addition, no risk factors for cholecystitis were identified, such as alcohol consumption or evidence of cholelithiasis. With the identification of the eschar during nursing care, scrub typhus was suspected and doxycycline was started, and the fever cleared within two days. The patient was discharged from hospital five days later, once the abdominal pain had completely subsided. We were unable to obtain follow-up images showing resolution of gallbladder wall edema or a clinical specimen for molecular confirmation of O. tsutsugamushi, due to the retrospective nature of this report.

Interestingly, EBV was also detected from serology. The patients showed several features consistent with primary mononucleosis, including tonsillopharyngitis, adenopathy, hepatosplenomegaly, and lymphocytosis. In addition, no eruption of rash was identified in this case, as previously described [61] We could not determine the percentage increase in atypical lymphocytes, which would have helped distinguish primary mononucleosis from reactivation [62]. Nevertheless, others have also reported mononucleosis-like features in scrub typhus [63]. As mentioned in the Introduction, EBV infection can also complicate and manifest as acute cholecystitis without lithiasis. During the lytic phase, the virus can replicate in tonsillar tissues and hepatocytes and manifest with splenomegaly. We wondered whether $O$. tsutsugamushi could trigger the activation of latent EBV and whether reactivation of B-lymphotrophic oncogenic viruses like EBV pose a risk for the development of malignancies [64].

Scrub typhus is a neglected tropical disease and a public health concern. Increasing public awareness about prevention and educating clinicians beginning work in endemic regions about scrub typhus, among other common tropical diseases, could help detect cases, start treatment in a timely manner, and prevent complications and death[65].

In conclusion, scrub typhus can complicate and manifest as acute acalculous cholecystitis in young adults. We therefore recommend using doxycycline empirically in cases presenting from regions endemic for scrub typhus, providing no contraindications are present.

Supplementary Materials: The following are available online at www.mdpi.com/xxx/s1: Figure S1. Timeline depicting the clinical course prior to hospitalization; Figure S2. Past and recent epidemiology of scrub typhus in atolls of the Maldives; Figure S3. Two decades of sentinel reporting of scrub typhus in the Maldives; Figure S4. Eschar lesion on the medial aspect of the thigh; and Figure S5. Clinical course during hospitalization.

Author Contributions: Conceptualization, A.A.A., H.A.I., W.N., E.E.N. and T.S.; methodology, H.A.I., W.N., W.M., E.E.N., and T.S.; software, H.A.I.; validation A.A.A., M.N., R.G., A.U., A.M., S.A.D., R.K.D., A.I.H., A.H., I.A., W.M., W.N., E.E.N. and T.S.; formal analysis, H.A.I., A.A.A., M.N., R.G., A.U., A.M., S.A.D., R.K.D., A.I.H., A.H., I.A., W.M., W.N., E.E.N. and T.S.; investigation, H.A.I., A.A.A., M.N., A.M., resources, A.A.A., M.N., R.G., A.U., A.M., S.A.D., R.K.D., A.I.H., A.H., I.A., W.M., W.N., E.E.N. and T.S.; data curation, H.A.I.; M.N., A.M., writing-original draft preparation, H.A.I., W.N., E.E.N. and T.S.; writing-review and editing, H.A.I., A.A.A., M.N., R.G., A.U., A.M., S.A.D., R.K.D., A.I.H., A.H., I.A., W.M., W.N., E.E.N. and T.S.; visualization, H.A.I.; supervision, A.U., W.N., E.E.N. and T.S.; project administration, H.A.I., A.A.A., M.N., R.G., A.U., A.M., S.A.D., R.K.D., A.I.H., A.H., I.A., W.M., W.N., E.E.N. and T.S. All authors have read and agreed to the published version of the manuscript.

Funding: This study was funded by the Japan Agency for Medical Research and Development (AMED, grant numbers JP19m0108003 and 21wm0225010h0102). In addition, this work was supported by International Postdoctoral Fellowship 2021, Mahidol University through W.N. and H.A.I.

Institutional Review Board Statement: The reporting of this case was conducted in accordance with the guidelines of the Declaration of Helsinki. An exemption to ethical approval was granted on September 29, 2021 (Ref: 137-B(NHA)/MISC/2021/045) by the National Healthcare Academy at Indira Gandhi Memorial Hospital, Malé, Republic of Maldives. 
Informed Consent Statement: Informed consent was obtained from the patient involved in the study.

Data Availability Statement: The data presented in this study are available on request from the corresponding author. The data are not publicly available to ensure the privacy of the study participant.

Acknowledgments: We are sincerely grateful to all staff at the Department of Surgery, particularly the wonderful nurses and surgical officers who cared for this patient. In addition, we are thankful to the Director of Medical Services, Dr. Shan Saeed, and Chief Executive Officer, Mr. Ibrahim Saleem, at Indira Gandhi Memorial Hospital. The authors would like to express their deepest gratitude to Dr. Jetsumon Prachumsri, Mahidol Vivax Research Unit, Faculty of Tropical Medicine, Mahidol University, for her administrative support, guidance, and constant encouragement.

Conflicts of Interest: The authors declare no conflicts of interest.

\section{References}

1. $\quad$ Paris DH. Special Issue "The Past and Present Threat of Rickettsial Diseases". Trop Med Infect Dis. 2020; 5.

2. Kelly DJ, Fuerst PA, Richards AL. Origins, Importance and Genetic Stability of the Prototype Strains Gilliam, Karp and Kato of Orientia tsutsugamushi. Trop Med Infect Dis. 2019; 4.

3. Richards AL, Jiang J. Scrub Typhus: Historic Perspective and Current Status of the Worldwide Presence of Orientia Species. Trop Med Infect Dis. 2020; 5.

4. Nagayo M, Miyagawa Y, Mitamura T, Imamura A. On the Nymph and Prosopon of the Tsutsugamushi, Leptotrombidium Akamushi, N. Sp. (Trombidium Akamushi Brumpt), Carrier of the Tsutsugamushi Disease. J Exp Med. 1917; 25:255-72.

5. Imad HA, Tanyaratsrisakul S, Piyaphanee W, Wattanagoon Y. Skin lesion from Maldives: Classic but forgotten. Travel Med Infect Dis. 2017; 17:74-5.

6. Hase T, Roberts LW, Hildebrandt PK, Cavanaugh DC. Stylostome formation by Leptotrombidium mites (Acari: Trombiculidae). J Parasitol. 1978; 64:712-8.

7. Megaw JW. Scrub Typhus as a War Disease. Br Med J. 1945; 2:109-12.

8. Philip CB. Tsutsugamushi disease in World War II. J Parasitol. 1948; 34:169-91.

9. Luce-Fedrow A, Lehman ML, Kelly DJ, Mullins K, Maina AN, Stewart RL, et al. A Review of Scrub Typhus (Orientia tsutsugamushi and Related Organisms): Then, Now, and Tomorrow. Trop Med Infect Dis. 2018; 3.

10. Giles HM, Symington T. Chloromycetin in scrub-typhus. Lancet. 1950; 1:16-9.

11. Weitzel T, Silva-de la Fuente MC, Martinez-Valdebenito C, Stekolnikov AA, Perez C, Perez R, et al. Novel vector of scrub typhus in subantarctic Chile - evidence from human exposure. Clin Infect Dis. 2021.

12. Izzard L, Fuller A, Blacksell SD, Paris DH, Richards AL, Aukkanit N, et al. Isolation of a novel Orientia species (O. chuto sp. nov.) from a patient infected in Dubai. J Clin Microbiol. 2010; 48:4404-9.

13. Ghorbani RP, Ghorbani AJ, Jain MK, Walker DH. A case of scrub typhus probably acquired in Africa. Clin Infect Dis. 1997; 25:1473-4.

14. Osuga K, Kimura M, Goto H, Shimada K, Suto T. A case of Tsutsugamushi disease probably contracted in Africa. Eur J Clin Microbiol Infect Dis. 1991; 10:95-6.

15. Horton KC, Jiang J, Maina A, Dueger E, Zayed A, Ahmed AA, et al. Evidence of Rickettsia and Orientia Infections Among Abattoir Workers in Djibouti. Am J Trop Med Hyg. 2016; 95:462-5.

16. Thiga JW, Mutai BK, Eyako WK, Ng'ang'a Z, Jiang J, Richards AL, et al. High seroprevalence of antibodies against spotted fever and scrub typhus bacteria in patients with febrile Illness, Kenya. Emerg Infect Dis. 2015; 21:688-91.

17. Masakhwe C, Linsuwanon P, Kimita G, Mutai B, Leepitakrat S, Yalwala S, et al. Identification and Characterization of Orientia chuto in Trombiculid Chigger Mites Collected from Wild Rodents in Kenya. J Clin Microbiol. 2018; 56.

18. Yen TY, Zhang Z, Chao CC, Ching WM, Shu PY, Tseng LF, et al. Serologic Evidence for Orientia Exposure in the Democratic Republic of Sao Tome and Principe. Vector Borne Zoonotic Dis. 2019; 19:821-7.

19. Lewis MD, Yousuf AA, Lerdthusnee K, Razee A, Chandranoi K, Jones JW. Scrub typhus reemergence in the Maldives. Emerg Infect Dis. 2003; 9:1638-41.

20. Scrub typhus surveillance data. Health Protection Agency, Ministry of Health, Maldives. 2021.

21. Hilmy AI, Dey RK, Imad HA, Yoosuf AA, Nazeem A, Latheef AA. Coronavirus disease 2019 and dengue: two case reports. J Med Case Rep. 2021; 15:171.

22. Miqdhaadh A, Imad HA, Fazeena A, Ngamprasertchai T, Nguitragool W, Nakayama EE, et al. Multisystem Inflammatory Syndrome Associated with SARS-CoV-2 Infection in an Adult: A Case Report from the Maldives. Trop Med Infect Dis. 2021; 6.

23. Brummaier T, Kittitrakul C, Choovichian V, Lawpoolsri S, Namaik-Larp C, Wattanagoon Y. Clinical manifestations and treatment outcomes of scrub typhus in a rural health care facility on the Thailand-Myanmar border. J Infect Dev Ctries. 2017; 11:407-13.

24. Rajapakse S, Weeratunga P, Sivayoganathan S, Fernando SD. Clinical manifestations of scrub typhus. Trans R Soc Trop Med Hyg. 2017; 111:43-54. 
25. Walsh K, Goutos I, Dheansa B. Acute Acalculous Cholecystitis in Burns: A Review. J Burn Care Res. 2018; 39:724-8.

26. Hu S, Dong Z, Wang C, Yang W, Chinese O, Metabolic Surgery C. Acute Gangrenous Acalculous Cholecystitis After Laparoscopic Roux-en-Y Gastric Bypass: a Case Report. Obes Surg. 2021.

27. Shapiro MJ, Luchtefeld WB, Kurzweil S, Kaminski DL, Durham RM, Mazuski JE. Acute acalculous cholecystitis in the critically ill. Am Surg. 1994; 60:335-9.

28. McChesney JA, Northup PG, Bickston SJ. Acute acalculous cholecystitis associated with systemic sepsis and visceral arterial hypoperfusion: a case series and review of pathophysiology. Dig Dis Sci. 2003; 48:1960-7.

29. Markaki I, Konsoula A, Markaki L, Spernovasilis N, Papadakis M. Acute acalculous cholecystitis due to infectious causes. World J Clin Cases. 2021; 9:6674-85.

30. Reed AC. Scrub Typhus. Cal West Med. 1944; 61:62-3.

31. Wang NC, Ni YH, Peng MY, Chang FY. Acute acalculous cholecystitis and pancreatitis in a patient with concomitant leptospirosis and scrub typhus. J Microbiol Immunol Infect. 2003; 36:285-7.

32. Hayakawa K, Oki M, Moriya Y, Mizuma A, Ohnuki Y, Yanagi H, et al. A case of scrub typhus with acalculous cholecystitis, aseptic meningitis and mononeuritis multiplex. J Med Microbiol. 2012; 61:291-4.

33. Lee H, Ji M, Hwang JH, Lee JY, Lee JH, Chung KM, et al. Acute Cholecystitis in Patients with Scrub Typhus. J Korean Med Sci. 2015; 30:1698-700.

34. Lee SJ, Cho YH, Lee SY, Jeong DW, Choi EJ, Kim YJ, et al. A case of scrub typhus complicated by acute calculous cholecystitis. Korean J Fam Med. 2012; 33:243-6.

35. Charoenphak S, Rattanawong P, Sungkanuparph S. Acute Cholecystitis as an Unusual Presentation of Scrub Typhus: A Report of Two Cases and Review of the Literature. Southeast Asian J Trop Med Public Health. 2017; 48:143-9.

36. Acharya S, Yadav JK, Khanal N, Bhandari R, Ghimire B. Acute Severe Calculous Cholecystitis with Multiorgan Failure Complicated by Scrub Typhus. Case Rep Surg. 2019; 2019:7505108.

37. Gagneux-Brunon A, Suy F, Pouvaret A, Pillet S, Tarantino E, Bouchet D, et al. Acute acalculous cholecystitis, a rare complication of Epstein-Barr virus primary infection: report of two cases and review. J Clin Virol. 2014; 61:173-5.

38. Aydin Teke T, Tanir G, Ozel A, Timur OM, Eksioglu AS. A case of acute acalculous cholecystitis during the course of reactive Epstein-Barr virus infection. Turk J Gastroenterol. 2013; 24:571-2.

39. Vermaak JS. Epstein-Barr virus acute acalculous cholecystitis. CMAJ. 2021; 193:E1143.

40. Harvey KG, Tice JG, Sigal A. Epstein-Barr Virus Causing Clinical Jaundice and Acute Acalculous Cholecystitis in a Previously Healthy 17-Year-Old Girl. Am J Case Rep. 2021; 22:e932285.

41. Nakagawa H, Miyata Y. Epstein-Barr virus infection associated with acute acalculous cholecystitis in a 20-year-old woman. CMAJ. 2021; 193:E696.

42. Audy JR. A summary topographical account of scrub typhus 1908-1946. In: Bulletins from the Institute for Medical Research, Federation of Malaya. Government Press Kuala Lumpur, Malaysia: 1949. 1949; 1.

43. Radford CD. Larval and nymphal mites (Acarina; Trombiculidae) from Ceylon and the Maldive Islands. Parasitology. 1946; 37:46-54.

44. Kalra SL. Progress in the knowledge of rickettsial diseases in India. Indian J Med Res. 1959; 47:477-83.

45. Wolstenholme RJ. The disease spectrum in a Maldivian (Adduan) population. Trans R Soc Trop Med Hyg. 1984; 78:505-7.

46. Kang N, Lan N, Ye H. Clinical analysis of tsutsugamushi disease misdiagnosed as tonsillitis. Lin Chung Er Bi Yan Hou Tou Jing Wai Ke Za Zhi. 2014; 28:425-6.

47. Atwal S, Giengkam S, Chaemchuen S, Dorling J, Kosaisawe N, VanNieuwenhze M, et al. Evidence for a peptidoglycan-like structure in Orientia tsutsugamushi. Mol Microbiol. 2017; 105:440-52.

48. Salje J. Orientia tsutsugamushi: A neglected but fascinating obligate intracellular bacterial pathogen. PLoS Pathog. 2017; 13:e1006657.

49. Bora T, Khan SA. Evaluation of Th1 and Th2 immune response in clinical and sub-clinical scrub typhus infection. Hum Immunol. 2019; 80:503-9.

50. Tantibhedhyangkul W, Ben Amara A, Textoris J, Gorvel L, Ghigo E, Capo C, et al. Orientia tsutsugamushi, the causative agent of scrub typhus, induces an inflammatory program in human macrophages. Microb Pathog. 2013; 55:55-63.

51. Kim MJ, Kim MK, Kang JS. Orientia tsutsugamushi inhibits tumor necrosis factor alpha production by inducing interleukin 10 secretion in murine macrophages. Microb Pathog. 2006; 40:1-7.

52. Min CK, Kim HI, Ha NY, Kim Y, Kwon EK, Yen NTH, et al. A Type I Interferon and IL-10 Induced by Orientia tsutsugamushi Infection Suppresses Antigen-Specific T Cells and Their Memory Responses. Front Immunol. 2018; 9:2022.

53. Lee JH, Lee JH, Chung KM, Kim ES, Kwak YG, Moon C, et al. Dynamics of clinical symptoms in patients with scrub typhus. Jpn J Infect Dis. 2013; 66:155-7.

54. Aung T, Supanaranond W, Phumiratanaprapin W, Phonrat B, Chinprasatsak S, Ratanajaratroj N. Gastrointestinal manifestations of septic patients with scrub typhus in Maharat Nakhon Ratchasima Hospital. Southeast Asian J Trop Med Public Health. 2004; 35:845-51.

55. Lee J, Kim DM, Yun NR, Kim YD, Park CG, Kim MW. The Correlation of Endoscopic Findings and Clinical Features in Korean Patients with Scrub Typhus: A Cohort Study. PLoS One. 2016; 11:e0155810.

56. Moron CG, Popov VL, Feng HM, Wear D, Walker DH. Identification of the target cells of Orientia tsutsugamushi in human cases of scrub typhus. Mod Pathol. 2001; 14:752-9. 
57. Lee Y, Kim SI, Yi YS, Lee H, Hwang JH, Park EC, et al. Transmission Electron Microscopy Confirmation of Orientia tsutsugamushi in Human Bile. Emerg Infect Dis. 2020; 26:3101-3.

58. Jeong YJ, Kim S, Wook YD, Lee JW, Kim KI, Lee SH. Scrub typhus: clinical, pathologic, and imaging findings. Radiographics. $2007 ; 27: 161-72$.

59. Keller CA, Hauptmann M, Kolbaum J, Gharaibeh M, Neumann M, Glatzel M, et al. Dissemination of Orientia tsutsugamushi and inflammatory responses in a murine model of scrub typhus. PLoS Negl Trop Dis. 2014; 8:e3064.

60. Yang CH, Young TG, Peng MY, Hsu GJ. Unusual presentation of acute abdomen in scrub typhus: a report of two cases. Zhonghua Yi Xue Za Zhi (Taipei). 1995; 55:401-4.

61. Akiyama Y, Ishikane M, Ohmagari N. Epstein-Barr virus induced skin rash in infectious mononucleosis. IDCases. 2021; 26:e01298.

62. Fedyanina OS, Filippova AE, Demina OI, Zhuliabina OA, Tikhomirov DS, Filatov AV, et al. The Nature and Clinical Significance of Atypical Mononuclear Cells in Infectious Mononucleosis Caused by the Epstein-Barr Virus in Children. J Infect Dis. 2021; 223:1699-706.

63. Watanabe Y, Mashimo S, Ichige H, Nagata H, Kojima M. Scrub typhus mimicking the clinical course of infectious mononucleosis: a case report. J Rural Med. 2021; 16:62-6.

64. Hjalgrim H, Friborg J, Melbye M. The epidemiology of EBV and its association with malignant disease. In: Arvin A, Campadelli-Fiume G, Mocarski E, Moore PS, Roizman B, Whitley R, et al., editors. Human Herpesviruses: Biology, Therapy, and Immunoprophylaxis. Cambridge2007.

65. Imad HA, Phadungsombat J, Nakayama EE, Suzuki K, Ibrahim AM, Afaa A, et al. Clinical Features of Acute Chikungunya Virus Infection in Children and Adults during an Outbreak in the Maldives. Am J Trop Med Hyg. 2021. 